\title{
Síntesis y caracterización de nuevas soluciones sólidas de fluoruros con estructura tipo fluorita y derivadas
}

\author{
V. ZAPATA-GALVÁN, L. M. TORRES-MARTÍNEZ Y P. QUINTANA* \\ Facultad de Ciencias Químicas, División de Estudios Superiores, Universidad Autónoma de Nuevo León, Monterrey, Nuevo León, México. \\ *Departamento de Física Aplicada, Cinvestav del IPN, Unidad Mérida, Mérida, Yucatán, México
}

\begin{abstract}
Se prepararon nuevas soluciones sólidas de fluoruros isovalentes con fórmula general $\mathrm{M}_{1-\mathrm{x}} \mathrm{Sn}_{\times} \mathrm{F}_{2}(\mathrm{M}=\mathrm{Ba}, \mathrm{Sr}, \mathrm{Cd})$ y $\mathrm{M}_{1 \times} \mathrm{Sn}_{1+\times} \mathrm{F}_{4}$ $(\mathrm{M}=\mathrm{Ba}, \mathrm{Sr})$. Las fases fueron sintetizadas por reacción en estado sólido en atmósfera de $\mathrm{N}_{2}$ y caracterizadas por difracción de rayos $\mathrm{x}$. La primera serie de compuestos son isoestructurales al $\beta-\mathrm{PbF}_{2}$, con simetría cúbica tipo fluorita. El intervalo de formación de las soluciones sólidas varía con el tamaño del ion siguiendo la secuencia $\mathrm{Ba}>\mathrm{Sr}=\mathrm{Cd}$. El valor de la celda unidad para el $\mathrm{Ba}$, disminuye con el contenido de $\mathrm{SnF}_{2}$, y para el Sr y Cd se mantiene constante. Los compuestos de la segunda serie, con mayor contenido de fluoruro de estaño, son isoestructurales al $\mathrm{PbSnF}_{4^{\prime}}$ con simetría tetragonal derivada de la fluorita ligeramente distorsionada.
\end{abstract}

Palabras clave: fluoruros, soluciones sólidas, fluorita, difracción de rayos $x$

Synthesis and characterization of new solid solutions with fluorite- and derivated structure type.

New solid solutions of isovalent fluorides, with chemical formula $\mathrm{M}_{1-\mathrm{S}} \mathrm{Sn}_{\mathrm{x}} \mathrm{F}_{2}(\mathrm{M}=\mathrm{Ba}, \mathrm{Sr}, \mathrm{Cd})$ and $\mathrm{M}_{1-x} \mathrm{Sn}_{1+x} \mathrm{~F}_{4}(\mathrm{M}=\mathrm{Ba}, \mathrm{Sr})$, were prepared. The phases were synthesised by solid state reaction under inert atmosphere and characterised by $x$-ray diffraction. The former compound series are isostructural to $\beta-\mathrm{PbF}_{2}$, with cubic symmetry fluorite type. The solid solutions range extension changes with the ion size, following the sequence $\mathrm{Ba}>\mathrm{Sr}=\mathrm{Cd}$. With $\mathrm{Ba}$, the unit cell diminishes with $\mathrm{SnF}_{2}$ content, and it maintains constant for $\mathrm{Sr}$ and $\mathrm{Cd}$. The latter compound series, with higher tin fluoride content, are isostructural to $\mathrm{PbSnF}_{4}$, a disorted fluorite with tetragonal symmetry.

Keywords: fluorides, solid solutions, fluorite, $x$-ray diffraction

*Trabajo presentado en la II Reunión Iberoamericana en Materiales Electrocerámicos (México, Abril 2000).

\section{INTRODUCCIÓN}

Desde los inicios de los años setenta, surgió un gran interés en el desarrollo de nuevos materiales conductores iónicos que pudiesen ser utilizados como electrolitos sólidos. Algunas de las aplicaciones tecnológicas más importantes son los sensores electroquímicos para la fabricación de celdas de combustible, de baterías; de sensores de gases para la detección de oxígeno en chimeneas industriales, en tubos de escape de los automóviles, para el monitoreo del funcionamiento de hornos y en la medición del contenido de oxígeno durante la producción de metales; en pantallas electrocrómicas, etc. $(1,2)$.

Algunos de los materiales que muestran ser prometedores electrolitos sólidos para la fabricación de sensores de oxígeno a temperatura ambiente son los halogenuros, en particular los fluoruros inorgánicos que presentan una estructura tipo fluorita y derivadas (3-8). Estos materiales son buenos aislantes electrónicos y dado que contienen vacantes disponibles, presentan una conductividad iónica rápida de iones $\mathrm{F}$ - Se ha encontrado que la movilidad del flúor se ve favorecida por la alta polarizabilidad de los cationes, especialmente cuando éstos tienen un par de electrones desapareados, y por la distribución catiónica desordenada que presentan los fluoruros con la presencia de cationes aliovalentes (4).

Varios compuestos con estructura tipo fluorita presentan valores elevados de conductividad del ion flúor (4,9-11). Esto se debe a que el átomo metálico forma una red cúbica centrada en las caras, y los átomos de flúor se encuentran ocupando todos los huecos tetraédricos; dejando por lo tanto, los huecos octaédricos desocupados, generando de esta manera una estructura bastante abierta que favorece la movilidad de los iones fluoruros $(12,13)$.

En general, estos materiales son considerados como electrolitos sólidos, siendo el $\mathrm{PbF}_{2}$ uno de los mejores ejemplos de conductor aniónico, estudiado por Faraday en 1834. El fluoruro de plomo es un conductor iónico pobre a $20^{\circ} \mathrm{C}$, sin embargo, al incrementar la temperatura pasa a ser un excelente conductor, $1 \mathrm{~S} / \mathrm{cm}$ a $400^{\circ} \mathrm{C}$. Se han observado tendencias similares en $\mathrm{CaF}_{2}, \mathrm{SrF}_{2}, \mathrm{BaF}_{2}$, aunque muestran una conductividad menor a temperaturas más elevadas (2). Las soluciones sólidas isovalentes y heterovalentes basados en $\mathrm{PbF}_{2}$, son materiales interesantes para desarrollar electrolitos sólidos con una conductividad rápida de iones fluoruros a moderadas y a bajas temperaturas, debido a que generan tanto vacantes de $\mathrm{F}^{-}$como iones intersticiales de $\mathrm{F}^{-}(11,14-16)$. El dopaje con iones isovalentes altamente polarizables como el $\mathrm{Sn}^{2+}$, ha mostrado un extraordinario incremento en la conductividad en los cristales de $\mathrm{PbF}_{2}$, formando un conductor rápido de aniones $\mathrm{PbSnF}_{4}$ (5-8). Sin embargo, presenta un esquema muy complicado de polimorfismo que depende de las condiciones de síntesis (16-18).

Los fluoruros de metales alcalino térreos han llamado la atención debido a que muestran una variedad de defectos en su estructura cristalina. Un dopaje adecuado del material, permite una distorsión en la estructura del fluoruro de origen, 
que dependiendo del tamaño del ion favorecen la conductividad $(10,19-21)$.

Por consiguiente, es nuestro interés preparar nuevos materiales no estequiométricos de fluoruros inorgánicos, con estructura tipo fluorita y relacionados, utilizando cationes bivalentes. En particular se presentará la síntesis y caracterización de los sistemas $\mathrm{MF}_{2}-\mathrm{SnF}_{2}$, donde $\mathrm{M}=\mathrm{Ba}$, $\mathrm{Sr}$ y Cd.

\section{PARTE EXPERIMENTAL}

La síntesis de las soluciones sólidas $\mathrm{M}_{1-\mathrm{x}} \mathrm{Sn}_{\mathrm{x}} \mathrm{F}_{2}(\mathrm{M}=\mathrm{Ba}$, $\mathrm{Sr}$, Cd) y $\mathrm{M}_{1-x} \mathrm{Sn}_{1+x} \mathrm{~F}_{4}(\mathrm{M}=\mathrm{Ba}$, Sr) se llevó a cabo por reacción en estado sólido. Se prepararon varias composiciones a partir

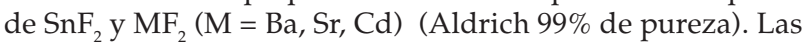
mezclas estequiométricas se homogeneizaron en acetona, moliéndolas mecánicamente en un mortero de ágata. Se prepararon pastillas que se cubrieron con polvo de la misma composición, para evitar la volatilización de los fluoruros. Estas se envolvieron con lámina de aluminio y se colocaron en navecillas de lámina de platino. Las muestras se sometieron a un tratamiento térmico inicial de $200^{\circ} \mathrm{C}$ y posteriormente se elevó la temperatura hasta $400-500^{\circ} \mathrm{C}$, en un período de 4-20 horas. Todos los tratamientos se realizaron en atmósfera inerte haciendo pasar un flujo de $\mathrm{N}_{2}$ en una mufla de tubo horizontal.

Para observar el desarrollo de la reacción se analizaron las pastillas periódicamente, por difracción de rayos $\mathrm{x}$ de polvos. Una vez que las mezclas mostraron una buena calidad, esto es, libres de líneas debidas a impurezas, se consideró que la reacción había terminado. El proceso de preparación se repitió con varias muestras asegurando de esta manera, la reproducibilidad de los resultados.

La identificación rutinaria de las soluciones sólidas se llevó a cabo por difracción de rayos x (Siemens D5000), utilizando radiación monocromática de $\mathrm{Cu}\left(\mathrm{K} \alpha_{1}=1.5405 \AA\right.$ ) , con un tiempo de contaje de $1.0 \mathrm{~s}$ por cada paso de $0.05^{\circ}$.

\section{RESULTADOS Y DISCUSIÓN}

Se prepararon mezclas seleccionando intervalos de $0.05 \%$ mol de $\mathrm{SnF}_{2}$, en los sistemas $\mathrm{MF}_{2}-\mathrm{SnF}_{2}$, donde $\mathrm{M}=\mathrm{Ba}$, $\mathrm{Sr}$ y Cd. Los productos de la reacción a diferentes tratamientos térmicos se encuentran en forma detallada en (22).

Para evitar la pérdida de material debido a la volatilización del fluoruro de estaño, se prepararon pastillas que fueron cubiertas con polvo de la misma composición. Tanto la pastilla como el polvo se envolvieron con lámina de aluminio y se colocaron en una navecilla de lámina de platino Las muestras se pesaron antes y después de cada reacción para comprobar que no hubiera pérdida de peso. Un problema que se presentó en algunas mezclas, fue la oxidación del estaño al término del tratamiento térmico (entre 300 y $400^{\circ} \mathrm{C}$ ), al sacar rápidamente las muestras de la mufla. Para evitar la formación de $\mathrm{SnO}_{2}$, se deslizó la navecilla de Pt hacia un extremo del tubo, para alejarla de la zona de calentamiento e iniciar un enfriamiento controlado, teniendo cuidado de mantener las pastillas en atmósfera de $\mathrm{N}_{2}$. De esta manera, fue posible evitar la oxidación del material mientras se enfriaban las muestras. Sin embargo, a temperaturas mayores de $500^{\circ} \mathrm{C}$, nuevamente se observó la formación de $\mathrm{SnO}_{2}$.

Las características de las dos nuevas series de soluciones sólidas se describen a continuación:

\subsection{Soluciones sólidas: $\mathrm{M}_{1-\mathrm{x}} \mathrm{Sn}_{\mathrm{x}} \mathrm{F}_{2}(\mathrm{M}=\mathrm{Ba}, \mathrm{Sr}, \mathrm{Cd})$}

Se observó la formación de una fase única con un patrón de difracción similar al compuesto de origen $\mathrm{MF}_{2}(\mathrm{M}=\mathrm{Ba}, \mathrm{Sr}$, Cd). No se detectaron cambios considerables en el patrón de difracción de rayos $\mathrm{x}$ al aumentar el contenido de $\mathrm{SnF}_{2}$, por lo tanto la existencia y la extensión de la solución sólida se determinó básicamente por la ausencia de reflexiones que pertenecieran a otra fase que no fuera $\mathrm{MF}_{2}$. Para corroborar la presencia de estaño en las soluciones sólidas se realizó por espectroscopía de electrones retrodispersados a bajo vacío (EDS), un microanálisis estadístico en diez zonas elegidas al azar. En la figura 1 se muestra el espectro para una muestra con baja concentración de fluoruro de estaño $\mathrm{Cd}_{0.975} \mathrm{Sn}_{0.025} \mathrm{~F}_{2}$.

Las soluciones sólidas tienen la fórmula general $\mathrm{M}_{1-\mathrm{x}}^{0.025} \mathrm{Sn}_{\mathrm{x}} \mathrm{F}_{2} \mathrm{y}$ son isoestructurales al compuesto $\beta-\mathrm{PbF}_{2}$, de estructura cúbica tipo fluorita. El fluoruro de estaño se incorpora a la estructura de la fluorita de manera sustitucional, de acuerdo con: $\mathrm{M}^{2+} \simeq \mathrm{Sn}^{2+}$. En la figura 2 se muestran los difractogramas de las tres soluciones sólidas, en concreto para la composición $x=0.05$, donde se observa el desplazamiento de las reflexiones hacia valores mayores de $2 \theta$, cuando se realiza la sustitución por un catión de menor radio iónico, siguiendo la siguiente secuencia $\mathrm{Ba}>\mathrm{Sr}>\mathrm{Cd}$.

Estas soluciones sólidas se formaron calentando las mezclas a partir de $200^{\circ} \mathrm{C}$ durante 3 horas y se mantuvieron estables al continuar el tratamiento térmico, ya sea aumentando el tiempo de calentamiento o incrementando la temperatura por períodos relativamente cortos. El intervalo de formación de las soluciones sólidas aumenta al elevar la temperatura, de tal manera que, para el caso del bario el intervalo es de $0<x<0.20$ a $200^{\circ} \mathrm{C}$ y aumenta a $\mathrm{x}=0.35$ a $500^{\circ} \mathrm{C}$. Para el estroncio la disolución del fluoruro de estaño varía desde 0.1 a $200^{\circ} \mathrm{C}$ hasta 0.15 a $400^{\circ} \mathrm{C}$. En el caso del cadmio, se mantiene constante en todo el intervalo de temperaturas, con un valor de $x=0.15$.

Para la determinación de los parámetros de la celda unidad, se registraron las muestras lentamente con un tiempo de contaje de $6 \mathrm{~s}$ por cada $0.01^{\circ}$. El cálculo de los parámetros se realizó en el intervalo de reflexiones de 5-90 $(2 \theta)$, con el método de Rietveld utilizando el programa GSAS. Los valores de los parámetros de red de las soluciones sólidas para el Ba son menores que los del fluoruro de origen $\mathrm{MF}_{2}(a=6.200 \AA$ ); a medida que aumenta el contenido de $\mathrm{SnF}_{2}$ el parámetro $a$ disminuye. Para el Sr y Cd se mantienen constantes (5.800 ̊̊ y $5.389 \AA$, respectivamente) (23). Estas diferencias se deben a la correlación entre los tamaños iónicos de los cationes que se reemplazan, para formar los fluoruros correspondientes. En la figura 3 se muestra la variación del parámetro $a$ con relación al radio catiónico medio: $r=r_{M} X_{M}+r_{S n} X_{S n^{\prime}}$ donde $r_{M}$ es el radio y $X_{M}$ la fracción del catión $\mathrm{M}^{2+}$.

\subsection{Soluciones sólidas: $\mathrm{M}_{1-\mathrm{x}} \mathrm{Sn}_{1+\mathrm{x}} \mathrm{F}_{4}(\mathrm{M}=\mathrm{Ba}$. $\mathrm{Sr})$}

En el caso de los sistemas con bario y estroncio, a mayor contenido de $\mathrm{SnF}_{2}$ se observa una región de coexistencia de dos fases: $\mathrm{M}_{\mathrm{x}} \mathrm{Sn}_{1-\mathrm{x}} \mathrm{F}_{2}$ y una serie de reflexiones cuyas características estructurales son muy similares al compuesto $\mathrm{MSnF}_{4}$. La estabilidad de la mezcla de las dos fases se mantiene en un intervalo estrecho de temperaturas, entre 200 y $400^{\circ} \mathrm{C}$.

En las composiciones cercanas al $50 \% \mathrm{~mol} \mathrm{de} \mathrm{SnF}_{2}$, se observó la formación de una nueva región estrecha de soluciones sólidas, estables entre 300 y $500^{\circ} \mathrm{C}$. Estas soluciones sólidas son isoestructurales con el $\beta-\mathrm{PbSnF}_{4}$ y tienen la fórmula gene- 


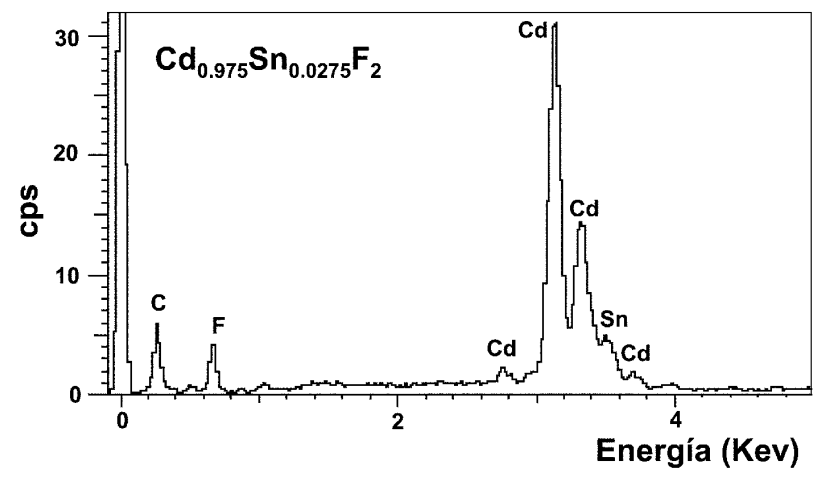

Fig. 1. Espectro EDS que muestra el análisis elemental para la composición $\mathrm{Cd}_{0.975} \mathrm{Sn}_{0.025} \mathrm{~F}_{2}$.

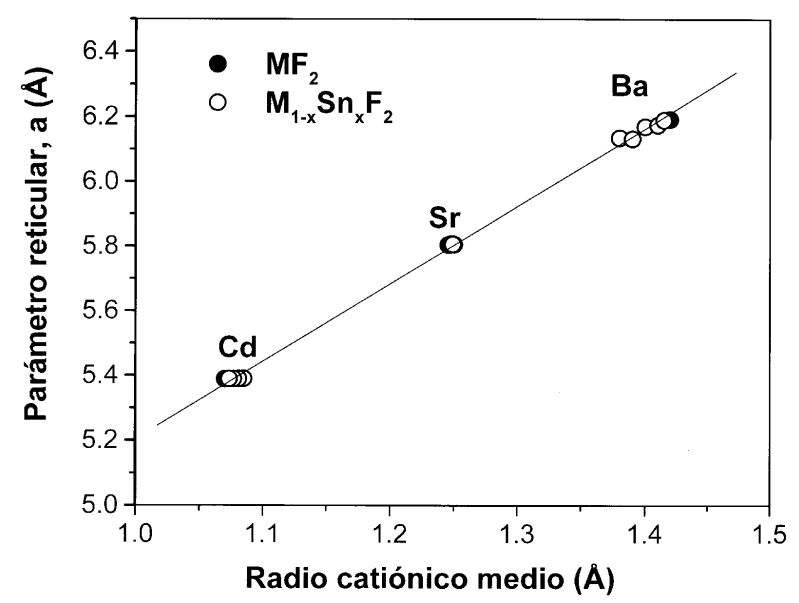

Fig. 3 Valores de los parámetros de red de la fase cúbica en función del radio catiónico medio para $\mathrm{M}_{1-\mathrm{x}} \mathrm{Sn}_{\mathrm{x}} \mathrm{F}_{2}(\mathrm{M}=\mathrm{Ba}, \mathrm{Sr}, \mathrm{Cd})$.

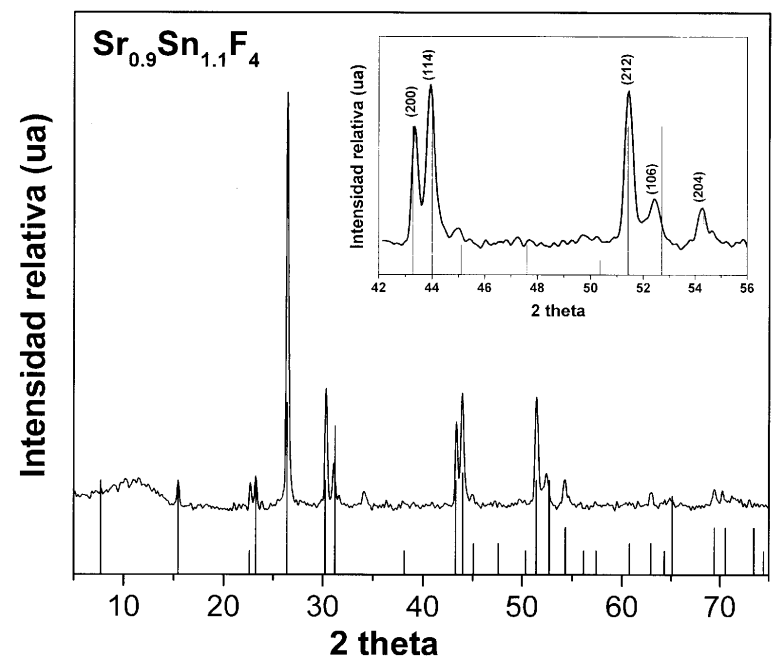

Fig. 5 Difractograma de $\mathrm{Sr}_{0.9} \mathrm{Sn}_{1.1} \mathrm{~F}_{4}$. En la parte inferior se muestran las líneas que corresponden al patrón de difracción de $\mathrm{SrSnF}_{4}(24)$.

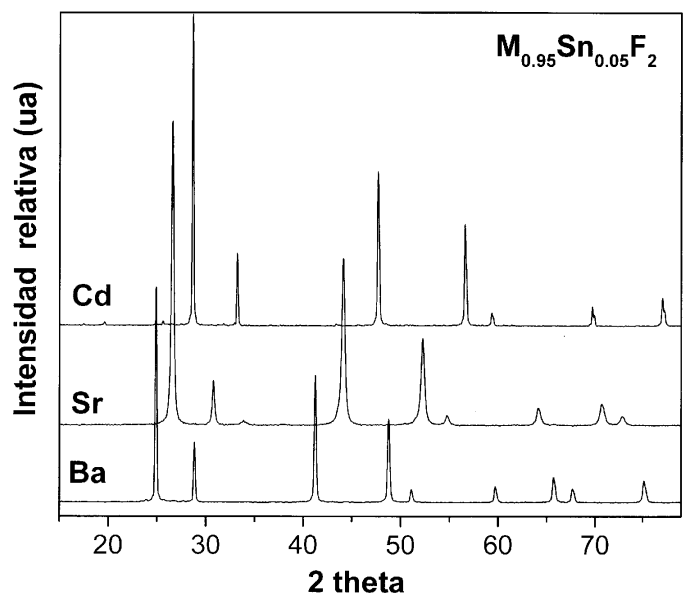

Fig. 2 Comparación entre los difractogramas correspondientes a las soluciones sólidas de tipo $\mathrm{M}_{1-\mathrm{x}} \mathrm{Sn}_{\mathrm{x}} \mathrm{F}_{2}(\mathrm{M}=\mathrm{Ba}, \mathrm{Sr}, \mathrm{Cd})$, para $\mathrm{x}=0.05$.

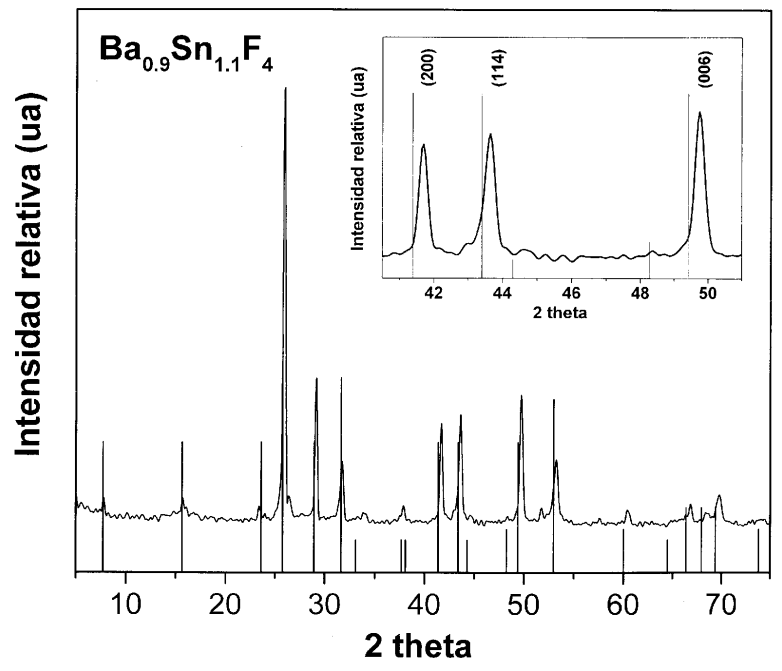

Fig. 4 Difractograma de $\mathrm{Ba}_{09} \mathrm{Sn}_{11} \mathrm{~F}_{4}$. En la parte inferior se muestran las líneas que corresponden al patrón de difracción $\mathrm{BaSnF}_{4}(24)$.

ral $\mathrm{M}_{1-\mathrm{x}} \mathrm{Sn}_{1+\mathrm{x}} \mathrm{F}_{4^{\prime}}$ donde $0<\mathrm{x}<0.11$. La estructura del $\mathrm{PbSnF}_{4}$ es una forma derivada de $\beta-\mathrm{PbF}_{2}$, la secuencia de la subred catiónica a lo largo del eje c es: $\mathrm{Pb} \mathrm{Pb} \mathrm{Sn} \mathrm{Sn} \mathrm{Pb} \mathrm{Pb} \mathrm{.....,} \mathrm{y} \mathrm{forma}$ una supercelda, duplicando el valor de $c$, por lo tanto se destruye la red de Bravais $\mathrm{F}$ y se rompe la simetría cúbica para dar lugar a una celda tetragonal $(4,18)$.

Si se comparan los difractogramas de las soluciones sólidas de bario y estroncio con los compuestos de los fluoruros mixtos $\mathrm{BaSnF}_{4}$ y $\mathrm{SrSnF}_{4}(24)$, se observa que las reflexiones se desplazan hacia la derecha, como se muestra en el recuadro de las figuras 4 y 5 , respectivamente. Los valores de los parámetros de red para $\mathrm{M}_{0.1} \mathrm{Sn}_{1.1} \mathrm{~F}_{4}$ son menores en comparación con los del fluoruro de origen, señalados entre los paréntesis: para el bario son $a=4.0503$ y $c=10.8596 \AA\left(\mathrm{BaSnF}_{4}, a=4.3564\right.$ y $c=$ $11.289 \AA$ A); y para el estroncio $a=3.7644$ y $c=10.3231 \AA$ $\left(\mathrm{SrSnF}_{4^{\prime}} a=4.1754\right.$ y $c=11.448 \AA$ ). 
En el caso del cadmio, no se observó la solución sólida debido a la formación de nuevas fases denominadas $\mathrm{G}$ y A, las cuales no pudieron ser identificadas con los patrones publicados en la literatura.

\section{CONCLUSIONES}

Se estudiaron y caracterizaron nuevas soluciones sólidas $\mathrm{M}_{1-\mathrm{x}} \mathrm{Sn}_{\mathrm{x}} \mathrm{F}_{2}(\mathrm{M}=\mathrm{Ba}, \mathrm{Sr}, \mathrm{Cd})$ que adoptan la estructura tipo fluorita. El límite de la solubilidad aumenta con el tamaño del radio iónico siguiendo la siguiente secuencia $\mathrm{Ba}>\mathrm{Sr}=\mathrm{Cd}$, en el intervalo de composiciones $0<x<0.35$ a $400^{\circ} \mathrm{C}, 0<\mathrm{x}<0.15$ a $400^{\circ} \mathrm{C}$ y $0<\mathrm{x}<0.15$ a $300^{\circ} \mathrm{C}$, respectivamente. En el caso de las soluciones sólidas de $\mathrm{Ba}$, el parámetro de la celda unidad, $a$, disminuye con la adición de $\mathrm{SnF}_{2}$.

Con mayor contenido de $\mathrm{SnF}_{2}$ se formó otra serie de soluciones sólidas de fórmula general: $\mathrm{M}_{1-\mathrm{x}} \mathrm{Sn}_{1+\mathrm{x}} \mathrm{F}_{4}$ donde $\mathrm{M}=\mathrm{Ba}$, $\mathrm{Sr}$, en el intervalo de composiciones $0<\mathrm{x}<0.11$ a $400^{\circ} \mathrm{C}$. Estos materiales son isoestructurales con el $\mathrm{B}-\mathrm{PbSnF}_{4}$ y tienen una simetría tetragonal derivada de la fluorita ligeramente distorsionada. Los valores de los parámetros de la red $a$ y $c$ son menores comparados con las muestras de origen, esto es con $\mathrm{x}=0$.

\section{AGRADECIMIENTOS}

Se agradece al CONACYT por el apoyo económico para la realización de este trabajo, mediante la beca otorgada a V. Zapata-Galván (3824P-A9607) y por la Cátedra Patrimonial de Excelencia Nivel II a P. Quintana (SC-970030-R98). Asímismo, se agradece a PAICYT-UANL (CA061-98); Red CYTED-VIIIF, a D. H. Aguilar por la asistencia técnica y a A.I. Puente Lara, de la Facultad de Química, UNAM, por haber realizado el análisis elemental por EDS.

\section{BIBLIOGRAFÍA}

1. C.A. Vincent, F. Bonino, M. Lazzari y B. Scrosati, “Modern Batteries. An Introduction to Electrochemical Power Sources" Edward Arnold (1985).

2. P.G. Bruce, "Solid State Electrochemistry", Cambridge University Press, Great Britain, (1997).

3. C. Lucat, J. Portier, J.M. Reau, P. Hagenmuller, “Etude par diffraction de neutrons de la solution solide $\mathrm{Pb}_{1-\mathrm{x}} \mathrm{Bi}_{2+\mathrm{x}}$ : Correlations entre structure et conductivité ionique". J. Solid State Chem. 32, 279-287 (1980).
4. P. Hagenmuller, "Inorganic Solid Fluorides-Chemistry and Physics", Academic Press, London p. 259, p. 423 (1985).

5. J. Kuwano, M. Asano, K., Shigehara y M. Kato, "Ambient temperature solidstate oxygen sensor using fast ion conductors, $\mathrm{PbSnF}_{4}$ and $\mathrm{Ag}_{6} \mathrm{I}_{4} \mathrm{WO}_{4}$ ". Solid State Ionics, 40/41, 472-475 (1990).

6. A. Wakagi, J. Kuwano, “Amperometric $\mathrm{PbSnF}_{4}$-based oxygen sensors: Rapid response at room temperature in the operating pressure range $10 \mathrm{kPa}-$ 7.2Mpa. J. Mater. Chem., 4, 973-975 (1994).

7. T. Eguchi, J. Kuwano. "Towards a room temperature, solid state, oxygen gas sensor". Mat. Res. Bull., 30, 1351-1357 (1995).

8. T. Eguchi, S. Suda, H. Amasaki, J. Kuwano, Y. Saito, "Optimum design for the sensing electrode mixtures of $\mathrm{PbSnF}_{4}$-based oxygen sensors for fast response at ambient temperature". Solid State Ionics, 121, 235-243 (1999).

9. A.K. Ivanov-Shits, N.I. Sorokin, P.P. Fedorov, B.P. Sobolev, "Specific features of ion transport in nonstoichiometric $\mathrm{Sr}_{1-x} \mathrm{R}_{\mathrm{x}} \mathrm{F}_{2+\mathrm{x}}(\mathrm{R}=\mathrm{La}-\mathrm{Lu}, \mathrm{Y})$ with the fluorite-type structure". Solid State Ionics, 31, 253-268 (1989).

10. A.K. Ivanov-Shits, N.I. Sorokin, P.P. Fedorov, B.P. Sobolev, "Specific features of ionic transport in nonstoichiometric fluorite-type $\mathrm{Ca}_{1-\mathrm{x}} \mathrm{R}_{\mathrm{x}} \mathrm{F}_{2+\mathrm{x}}(\mathrm{R}=\mathrm{La}-\mathrm{Lu}$, Y, Sc) phases". Solid State Ionics, 37, 125-137 (1990).

11. V. Trnovcová, P. P. Fedorov, I.I. Buchinskaya, V. Smatko, F. Hanic, "Fast ionic conductivity of $\mathrm{PbF}_{2}: \mathrm{ScF}_{3}$ single crystals and composites". Solid State Ionics, 119 181-189 (1999)

12. B.P. Sobolev, "Non-Stoichiomestry in Inorganic Fluorides and Phases with Fluorite Structure". Butll. Soc. Cat. Cien, 12, 275-331 (1991).

13. Z.L. Wang, Z.C. Kang, "Functional and Smart Materials. Structural Evolution and Structure Analysis". Plenum Press, New York, p.151 (1998).

14. I. Kosacki, "Anion disordering and band structure of $\mathrm{PbF}_{2}$ superionic crystals". Solid State Ionics, 28-30, 449-451 (1988).

15. H. Podsiadlo, "Phase equilibria in the binary system lead fluoride $\left(\mathrm{PbF}_{2}\right)$ Cadmium Fluoride $\left(\mathrm{CdF}_{2}\right)$ ". J. Thermal Anal., 54, 863-866 (1998)

16. L. Garza Tovar, "Diagrama de Fases y Propiedades Eléctricas en el Sistema $\mathrm{PbF}_{2}-\mathrm{SnF}_{2}$ ", Tesis doctoral en Ingeniería de Materiales, UAEM (2001)

17. G. Denes, Y.H. Yu, T. Tyliszczak, A.P. Hitchcock, "Sn-K and Pb-L EXAFS, Xray diffraction, and ${ }^{119} \mathrm{Sn}$ Mössbauer spectroscopic studies of ordered $\beta$ $\mathrm{PbSnF}_{4}$ and disordered $\mathrm{Pb}_{1-x} \mathrm{Sn}_{x} \mathrm{~F}_{2}(\mathrm{x}=0.3,0.4)$ solid solutions and $\mathrm{PbSn}_{4} \mathrm{~F}_{10}$ : High performance fluoride ion conductors". J. Solid State Chem. 104, 239252 (1993).

18. A. Collins, G. Denes, D. Le Roux, M.C. Madamba, J. M. Parris, A. Salaün, "Understanding the phase transitions and texture in superionic $\mathrm{PbSnF}_{4}$. The key to reproducible properties". Int. J. Inorg. Mater.1, 289-301 (1999).

19. A. M. Golubev, A.K. Ivanov-Shits, V.I. Simonov, B. P. Sobolev, N.I. Sorokin, P.P. Fedorov, "A structural model for fluoride ionic transport in $\mathrm{Ba}_{1-x} \mathrm{Ho}_{x} \mathrm{~F}_{2+x}$ solid solutions ( $x \varnothing 0.1)$. Solid State Ionics, 37, 115-121 (1990).

20. S. N. Achary, S. Mahapatra, S.J. Patwe, A.K. Tyagi, "Synthesis and characterization of mixed fluorides $\mathrm{Y}_{1-\mathrm{x}} \mathrm{Ba}_{2+1.5} \mathrm{~F}_{7}(-1.33 \leq \mathrm{x} \leq 1.0)$. Mat. Res. Bull. 34, 1535-1543 (1999)

21. S. J., Patwe S. N. Achary, A. K. Tyagi, S. Mahapatra, "Synthesis and characterization of mixed fluorides $\mathrm{Y}_{1-\mathrm{x}} \mathrm{Ba}_{2+1.5 x} \mathrm{~F}_{7}(-1.0<\mathrm{x} 0.5)^{\prime \prime}$. Mater. Res. Bull. 34, 761-769 (1999).

22. V.A. Zapata Galván, Tesis de Maestría, Universidad Autónoma de Nuevo León, México, p. 49 (1999).

23. R.D. Shannon, C.T. Prewitt, "Effective ionic radii in oxides and fluorides". Acta Cryst. B25, 925-956 (1969).

24. Powder Diffraction File, International Center for Diffraction Data (ICDD, tarjetas 4-452, 6-262), Pennsylvania, USA (1998). 